\title{
Descentralización y gestión de los servicios de salud en el estado Aragua*
}

\author{
Rincón Becerra, María T.* \\ Rodríguez Colmenares, Isabel ${ }^{* \star *}$
}

\section{Resumen}

Con la descentralización político-territorial iniciada en Venezuela a fines de los ochenta, las gobernaciones como instancias receptoras de competencias emprenden un conjunto de reformas tanto en su política como en su administración. Este trabajo tiene como objetivo estudiar algunos modelos de gestión de los servicios de salud promovidos por la gobernación del Estado Aragua. La investigación es exploratoria, se fundamenta en documentos y entrevistas semiestructuradas realizadas a informantes clave, la muestra la constituyen el Hospital José María Vargas y la Fundación Hospital Los Samanes. Los resultados demuestran: 1) Procesos de decisión centralizados en la cúspide del poder; 2) Selección de personal bajo criterios meritocráticos; 3) Flexibilización y precarización laboral; 4) Incorporación de los usuarios en el financiamiento de los servicios de salud; 5) Predominio de una atención curativa en detrimento de la preventiva. Se concluye que la descentralización ha dado paso a la privatización de los servicios, en contraposición a los preceptos constitucionales.

Palabras clave: Servicios de Salud, toma de decisión, flexibilización, precarización laboral, privatización, descentralización, gobernaciones.

Recibido: 04-12-07. Aceptado: 05-02-21

* Este trabajo forma parte de los resultados del Proyecto de Investigación: "Modelos de gestión de los servicios de salud promovidos por las gobernaciones en Venezuela". Aprobado por el FONACIT en su Programa de Investigador Novel (PIN) para la formación de María Teresa Rincón, adscrito al Programa Política y Administración Subnacional del Centro de Estudios de la Empresa (CEE) de la Facultad de Ciencias Económicas y Sociales (FCES) de la Universidad del Zulia (LUZ).

** Lic. en Ciencia Política. Magíster en Dirección y Gestión Pública Local. Docente e Investigadora del CEE de la FCES de LUZ. Acreditada en el programa de Promoción al Investigador (PPI) del FONACIT. E-Mail: materinbe2@yahoo.com / materinbe@hotmail.com

*** Sociólogo. Magíster en Ciencia Política. Investigadora del CEE de la FCES de LUZ. Acreditada en el programa de Promoción al Investigador (PPI) del FONACIT. E-mail:isarodri@cantv.net. 


\section{Decentralization and health service management in Aragua State}

\section{Abstract}

Along with the political-territorial decentralization which began in Venezuela in the late 1980s the state governments initiated a series of political and administrative reforms. The objective of this paper is to study some of the health services management models promoted by the state government of Aragua State. The research is descriptive, field-oriented, analytical, and is based on documents and semistructured interviews applied to key informants. The sample population was the Jose Maria Vargas Hospital and the Los Samanes Foundation Hospital. The results indicate that: 1) decisionmaking processes are centralized at a high level, 2) the selection of personnel was based on merit criteria, 3) there was labor flexibility and precariousness, 4) the users of the services helped in financing the health services, and 5) the attention offered was more curative than preventative. The conclusion is that decentralization has opened the door to privatization of services, which is contrary to constitutional precepts.

Key words: Health service, decision-making, flexibilization, labor precariousness, private financing, decentralization, state government.

\section{Introducción}

La descentralización en materia de salud, se inicia en 1990, en el marco de aprobación de la Ley Orgánica de Descentralización, Delimitación y Transferencia de Competencias del Poder Público (LODDT), (Congreso de la República, 1989), y la Ley Orgánica de Elección y Remoción de los Gobernadores y Alcaldes, con lo cual las gobernaciones se convierten en entidades receptoras de competencias. Los recién electos gobernadores solicitan la transferencia de los servicios de salud, la cual comienza a ser efectiva a partir de 1993. Este proceso se lleva a cabo sin que el
Ministerio de Sanidad y Asistencia Social (MSAS) ${ }^{1}$ realizara previamente una evaluación para determinar si las gobernaciones como entes receptores de las nuevas responsabilidades contaban 0 no con las capacidades suficientes para asumir el nuevo rol a desempeñar (Rincón, 2004). En este sentido, las gobernaciones cuando asumieron el proceso de descentralización comenzaron a definir; no solamente las acciones a seguir, sino también enfrentaron el reto de estructurar modalidades de gestión para la prestación de los servicios de salud.

En este trabajo se exploran dos modelos de gestión de los servicios de salud pública implementados por la gobernación

1 En el año 1999 este ministerio sufre un proceso de reforma integral, dada la fusión entre dicho ministerio y el de familia pasándose a llamar Ministerio de Salud y Desarrollo Social (MSDS). 
del Estado Aragua ${ }^{2}$ (Venezuela). Partimos del supuesto que la descentralización, ha dado paso a la implementación de diversas formas de gestión en materia de salud pública. Siguiendo los planteamientos de la propuesta Burocrática de Weber (1976), el modelo burocrático populista (Ochoa et al., 1996), el Tecnocrático (Ochoa, 1995) y la Nueva Gestión Pública (CLAD, 1998), centramos la atención en las siguientes variables objeto de estudio: toma de decisión, administración de personal, prestación de los servicios de salud $y$ fuentes de financiamiento.

La investigación es exploratoria, el criterio de selección del Estado Aragua se erige sobre la base de ser una entidad pionera a nivel nacional en adelantar acciones relacionadas con el sector salud. Se constituyó en la primera Región Sanitaria del país en 1947. En 1993 se impulsó el proceso de descentralización de los servicios de salud, transfiriéndose a la gobernación los establecimientos y programas del MSAS, creándose una estructura de transición como lo fue el Servicio Autónomo de Salud de Aragua (SASEA), que a partir de 1996 se transformó en un Instituto Autónomo con personalidad jurídica y patrimonio propio, denominado Corporación de la Salud del Estado Aragua (CORPOSALUD).

La muestra de estudio está conformada por el Hospital José María Vargas ${ }^{3}$, en Cagua, creado por el MSAS en 1972; y el Hospital Estadal Los Samanes ${ }^{4}$, fundado en 1989 coincidiendo con los inicios del proceso de descentralización. La muestra no requirió de una representatividad de elementos de una población, sino a una cuidadosa y controlada elección de sujetos con ciertas características especificas (Hernández et al., 1991).

\section{La descentralización de la salud en el Estado Aragua}

A inicios de la década de los noventa, el Estado Aragua registró una tasa de natalidad de 23.68 por mil nacidos vivos; mortalidad general de 4.28 por mil habitantes; mortalidad infantil de 27.73 por mil nacidos vivos; mortalidad temprana de 0.69 muertes por mil niños nacidos; morta-

El Estado Aragua limita por el norte con el Mar Caribe; al oeste con el Estado Miranda y el Distrito Metropolitano de Caracas; al oeste con el Estado Carabobo; $y$ al sur con el Estado Guárico. Esta conformado por 18 municipios. Cuenta con un población de 1.449 .616 habitantes, representando el 6.3\% de la población total del país. Tiene una extensión de 7.014 Km2. Sus principales actividades económicas son: industria, agropecuaria, pesca y turismo (SISOV, 2005).

3 Presta atención de nivel primario y secundario, cuenta con algunos servicios que corresponden al nivel terciario. Su área natural esta conformada por una población de alrededor de 100.000 habitantes, cuenta arquitectónicamente con 25 camas, de las cuales 20 están presupuestadas y en funcionamiento. Se realizan actividades docentes de pre y postgrado.

4 La atención prestada es ambulatoria de nivel primario y secundario. Es el principal Hospital Materno Infantil con que cuenta éste Estado. En 1992 inaugura el servicio de Hospitalización Pediátrica con 70 camas estando presupuestadas hasta el 2003 un total de 25 camas. A partir de 1995, se constituye en el asiento de la Fundación Ambulancias y Diagnósticos de Los Samanes; y se realizan actividades docentes de pre y postgrado. Cuenta con servicios de laboratorio, servicios de radiología, entre otros. 
lidad materna de 0.64 por mil partos. Entre las principales causas de morbilidad, resaltaron: faringoamigdalitis, diarreas, virosis e infecciones respiratorias agudas (Gobernación de Aragua, 1995: 7-10).

La capacidad instalada de establecimientos de salud del sector público fue de un $83 \%$ y del sector privado $13 \%$. Del porcentaje perteneciente al Estado, el MSAS concentraba el $72 \%$ de la red hospitalaria y ambulatoria; $4 \%$ el Instituto Venezolano de los Seguros Sociales (IVSS), y 7\% pertenecía al Instituto de Previsión y Asistencia Social del Ministerio de Educación (IPASME), Fuerzas Armadas Nacionales, Gobernación y Alcaldía de Girardot (Gobernación de Aragua, 1995).

Con la Ley de Descentralización y Transferencia de Competencias en 1990, el gobierno regional adelanta la descentralización mediante la negociación de un convenio con el MSAS, dando paso a la creación de la Comisión de Salud, la cual comenzó sus acciones con el Programa Núcleos de Atención Primaria en salud ${ }^{5}$.

El Gobernador Carlos Tablantes (1993), emprende el proyecto regional de salud basado en tres aspectos básicos de la descentralización: 1) acercar la salud a los usuarios y a la comunidad, lo que fue interpretado como la búsqueda del acceso de la población a la atención acorde a sus necesidades; 2) elevar la calidad del servicio; orientada a promover la accesibilidad, oportunidad, seguridad y racionalidad técnica con atención humanizada, de tal manera, que el usuario se sintiera satisfecho; y 3) adopción de un nuevo modelo de gerencia hospitalaria, basado en la autogestión ${ }^{6}$ y en la cogestión ${ }^{7}$, donde las personas de menos recursos no pagarían por el servicio.

Este proyecto dio paso a la venta de los servicios de salud, mediante la recuperación de costos, con la cual se fue modificando progresivamente el origen de los fondos de financiamiento (Gamboa, 2003). Esto evidencia el surgimiento de una alternativa de gestión que dio origen a una práctica privatizadora de los servicios de salud, que arrojó como consecuencia inmediata la exclusión de un grupo de la población, profundizando de esta manera las desigualdades.

En ese mismo año, la Comisión de Salud, mediante decreto regional pasa a convertirse en el Servicio Autónomo de Salud del Estado Aragua (SASEA), como ente regional dependiente de la gobernación, sin personalidad jurídica, con autonomía funcional en el campo financiero, presupuestario y de gestión; conformándose así en una estructura de transición entre el viejo modelo centralizado y el nuevo modelo descentralizado. El SASEA, se crea con el propósito de administrar los servicios del sistema de salud y

5 La Atención Primaria, nace formalmente en Venezuela a inicios de la década de los noventa y su primera operacionalización se concreta con la formación de los Núcleos de Atención Primaria.

6 Este término se han prestado a distintas interpretaciones no existiendo suficiente claridad; pero de acuerdo a la información recaba se interpretó que la autogestión connota, el financiamiento mediante el cobro de los servicios a los usuarios. 
formular planes y programas regionales (Gobernación del estado Aragua, 1995).

En 1996, la Asamblea Legislativa del Estado Aragua, aprueba la Ley de Salud, con el propósito de preservar, defender y restituir la salud de los habitantes del Estado, garantizándoles este derecho en su ámbito territorial, el desarrollo de la atención primaria y el saneamiento ambiental, como fundamentos básicos de la protección de la salud, con la finalidad de mejorar la calidad de vida de la población. Mediante esta Ley el SASEA es sustituido por CORPOSALUD, como Instituto Autónomo adscrito a la Gobernación del Estado, con personalidad jurídica, patrimonio y autonomía propia, y como ente descentralizado del Servicio Nacional de Salud, con rango de Secretaría, erigiéndose en la máxima autoridad sanitaria de la región (Gobernación del Estado Aragua, 1995).

Los lineamientos trazados por CORPOSALUD, apuntaron básicamente hacia: a) combatir las desigualdades de las personas ante los beneficios de la salud, la enfermedad y el acceso a los servicios; b) construcción un sistema de salud integral, accesible y de calidad que privilegiara la promoción de la salud y la prevención de la enfermedad; y, c) Fortalecer el papel rector de Corposalud a través de un sistema público de salud participativo, descentralizado y coordinado.

Para lograr dichos objetivos se comenzó a definir una estrategia que inte- graba todos los servicios y programas de salud públicos existentes, tanto a nivel de los municipios como de las parroquias, para organizarlos de acuerdo a la división político territorial existente, por lo que además de los cinco distritos sanitarios existentes, fueron creados tres nuevos para alcanzar un total de ocho: Maracay; La Victoria; Zamora; San Sebastián; Cagua; Mariño; Mario B. Irragorri y Palo Negro. En 1997, se mejora la capacidad técnica y administrativa de los mencionados distritos, con la finalidad de ir desconcentrando progresivamente las funciones y los recursos del nivel regional al distrital (Rodríguez, 2000: 322).

Para 1998, el gobierno regional profundiza el proceso de descentralización, transformando los distritos sanitarios en municipios sanitarios, en correspondencia con la división político territorial del Estado Aragua. Esta nueva configuración sanitaria cuenta con 8 hospitales generales; 2 especializados; 134 ambulatorios urbanos y 57 ambulatorios rurales. Adicionalmente, existen 2 ambulatorios del IPASME; 2 Hospitales del Instituto Nacional de Geriatría (INAGER); así como también 1 hospital del Ministerio de Defensa; 4 ambulatorios y 2 Hospitales del IVSS; para sumar un total de 212 establecimientos de Salud (Dirección Regional de Salud del Estado Aragua, 2000).

Además este Estado cuenta con los servicios relacionados con el INA-

Con la cogestión sucede algo similar, constituyendo la interpretación más difundida la que hace alusión a la participación de los trabajadores en la empresa sin que supongan alteración de las relaciones laborales; sin embargo, en este caso la cogestión fue interpretada como la búsqueda de la relación de compromiso del Estado con otros entes público u organizaciones de la sociedad para satisfacer las necesidades de salud. 
GER, el Instituto Nacional de Nutrición (INN); Programa Ampliado Materno Infantil (PAMI), la Fundación para el Mantenimiento de la Infraestructura Médico Asistencial (FIMA). Con estos programas se revelan las nuevas responsabilidades transferidas del Poder Central al gobierno subnacional, agilizando a nivel regional el proceso de decisión en materia de salud. Sin embargo, es de precisar que la descentralización, no ha contribuido a profundizar la democratización del sistema político, pues se ha coaccionado la participación de los ciudadanos en la toma de decisión.

Simultáneo al proceso de descentralización, el Estado Aragua adelanta el Modelo de Atención Integral (MAl); en este sentido, se toman decisiones orientadas a: 1) fortalecer y modernizar la red asistencial ambulatoria ${ }^{8}$; 2) relanzar los programas preventivos; y 3) constituir los Núcleos de Atención Primaria, que no es mas que generar la vinculación de los servicios de salud con la comunidad. El MAI se planteó dos objetivos: 1) integrar las consultas fragmentadas en una sola; provisionalmente, reducirlas a tres grandes actividades: atención integral al niño al adolescente $y$ al adulto en general; $y$ 2) crear una historia clínica con un mismo tipo de formato, para toda la red ambulatoria, con el propósito de generar un sistema que permitiera integrar la información (MSDS, 2001).

Este modelo constituyó un esfuerzo del gobierno regional en la búsqueda de transformar el modelo médico-asistencialista tradicional por uno orientado a la prevención y promoción, teniendo como base la atención primaria en salud. Esto condujo a que el MSDS, como ente rector de la política de salud, decretada en el 2000, la ejecución del MAI en todo el territorio nacional.

Asimismo, en el contexto de la descentralización también se emprendió con el apoyo técnico de la OMS y la OPS; la estrategia "Municipios Saludables", mejor conocida como Municipios hacia la Salud ${ }^{9}$ en Venezuela. La incorporación de los municipios a esta estrategia exigía primordialmente voluntad política de los gobernantes para implementarla. En el caso del Estado Aragua el municipio Urdaneta fue incorporado por ser el tercero más pobre de esta entidad regional, con un $55 \%$ de necesidades básicas insatisfechas.

Municipios saludables, se fundamentó en la promoción de la salud con el propósito de reducir la inequidad, enfatizando líneas de acción orientadas hacia la elaboración de políticas públicas saludables; creación de ambientes favorables; reforzamiento de la acción comunitaria; desarrollo de habilidades personales y cambios en los estilos de vida; reorientación de los servicios de salud; y oportunidades de vida. El compromiso de la alcaldesa municipal estuvo orientado al cumplimiento de estas seis líneas de acción, la cual se fundamentó en la planificación estratégica; por lo que se identifi-

8 Se trata de un conjunto coordinado de programas, servicios y niveles de salud.

9 Este proyecto es la adaptación del proyecto Ciudades Sanas que se realiza en Europa desde 1986. 
caron las áreas problemas y se elaboraron 8 proyectos de intervención para contrarrestar los efectos de las dificultades identificadas. Todo esto se llevó a cabo con la participación de los organismos internacionales y nacionales, así como con el apoyo de la comunidad (Málaga et al., 1996).

Sin ánimo de agotar el recorrido de la descentralización de los servicios de salud en el estado Aragua, nos planteamos más adelante destacar algunas particularidades de la gestión de los servicios de salud, en dos casos de estudio, precisando los rasgos que en la realidad se manifiestan en la prestación del servicio.

\section{Consideraciones teóricas sobre gestión del aparato público}

En esta sección del artículo se realiza una breve consideración teórica que permita destacar los rasgos que asumen procesos, tales como: toma de decisión, administración de personal, prestación y financiamiento de servicios en los modelos de gestión citados anteriormente y que discutiremos a continuación.

\subsection{Rasgos del modelo weberiano}

La burocracia como forma organizativa, descansa sobre la racionalidad en la adecuación de los medios a los fines pretendidos. El término fue utilizado por primera vez a mediados del siglo XVIII, y fue esgrimido para referirse al poder del cuerpo de funcionarios y empleados de la administración estatal constituido para tareas especializadas (Weber, 1976).

El modelo ideal de Burocracia Weberiano se inspira en la extrema división del trabajo, con grandes estructuras organizativas, numerosos niveles jerárquicos y departamentos, con clara asignación de tareas especializadas a cada trabajador (Weber, 1976). Estas grandes estructuras, conducen al establecimiento de una burocracia rígida y formal que afecta la toma de decisión al no permitir que estas se produzcan en una forma rápida y oportuna, ya que la autoridad por sus atribuciones impone una lógica de concentración de poder en la cúspide de la jerarquía, es decir, quien decide es siempre aquél que ocupa el puesto más alto. El proceso de decisión resulta poco flexible e impide el consenso y el compromiso de la mayoría. Ya que las decisiones son tomadas por la autoridad superior, ello confiere a las personas investidas de autoridad un poder de coacción sobre los subordinados.

Según Weber (1976: 718-722) el burócrata es seleccionado de acuerdo a sus méritos con base a pruebas especiales. La ocupación del cargo es considerada como la aceptación de un deber específico de fidelidad al mismo ${ }^{10}$, a cambio de la garantía de una existencia futura asegurada, ya que el funcionario tiene la perspectiva de ser ratificado o removido en el cargo por su jefe. El empleado en el desempeño de sus actividades y de acuerdo a la duración del tiempo de servi- 
cio, es retribuido con un sueldo fijo. El funcionario está ubicado en un escalafón, que le permite aspirar a: un ascenso en el cargo; en los salarios de acuerdo al tiempo de servicio prestado y a las necesidades de la organización; y mediante pruebas que demuestren sus conocimientos. Al llegar la vejez, los funcionarios aspiran que por el estatus alcanzado a lo largo de su estancia en la organización obtener una pensión de vejez.

La incapacidad de respuesta del Estado a las demandas sociales mediante la prestación y financiamiento de los servicios públicos, se fundamenta en la orientación de reglas y normas pretendidamente universales desarrolladas por la administración pública burocrática, lo que permitió la puesta en práctica de un modelo organizacional auto-referido, donde las acciones del Estado giraban en torno a su propia relegitimación del poder, y no hacia la satisfacción de las expectativas de los usuarios, los cuales a su vez fueron replegados y desmovilizados (CLAD, 1998).

\subsection{Rasgos del Modelo Burocrático Populista}

El modelo burocrático populista es un modelo administrativo que en lo prescriptivo se sustenta en Weber, cuenta con un conjunto de características propias. Para Ochoa et al., (1996: 41), lo definen como un "modelo administrativo público iniciado con la democracia, puesto que se impone una concepción de la condición del aparato público, basada en la búsque- da de colaboración de clases a través del gasto público para el logro de los objetivos".

La instauración de este modelo, se dió en el avance de la economía de sustitución de importaciones, y por el crecimiento de los partidos políticos (Ochoa et al., 1996), particularmente de partidos populistas caracterizados por una marcada inclinación hacia la personalización del liderazgo que favorece una innegable orientación autoritaria, en detrimento de un régimen orientado por valores éticos que permitieran un desarrollo diferente del aparato público. Se adelantó tanto un estilo de hacer política, que privilegiaba el ascenso a los puestos de la dirección estatal por la vía de la influencia partidista, como la captura de todo el aparato público por parte de los partidos políticos (Ramos, 1995).

La administración burocrática populista, se caracteriza por contar con grandes estructuras organizativas con altos niveles jerárquicos y numerosos departamentos propios del modelo Weberiano, lo que demuestra el desarrollo de procesos de decisión centralizados ${ }^{11}$; tanto las decisiones de tipo estratégicas como aquellas de tipo cotidiano son tomadas en la cúpula de la administración pública nacional (Ochoa et al., 1996).

Se desarrolla un proceso de toma de decisiones personalista, que se manifiesta con la influencia política y el escaso uso de canales formales; esto hace que en la trayectoria del flujo de la decisión se desvirtúe o se distorsione. Tienen mayor

11 La centralización de las decisiones tiene doble consecuencias, por un lado, dificulta la democratización del poder, y por otro, afecta negativamente la eficiencia (Ochoa et al, 1996). 
importancia los canales informales, tales como: la palanca, la recomendación, los contactos y los grandes cogollos. Este esquema, se orienta a complacer a todos, a no sacrificar a nadie, lo que retarda las decisiones imponiendo sacrificios, haciendo que los decisores prefieran, al mismo tiempo, políticas vistosas y populares capaces de generar amplias simpatías a corto plazo (Ochoa, 1996/1997).

La informalidad en la toma de decisiones provoca que a posteriori se haga difícil el seguimiento de la correlación entre la decisión y lo que se ejecutó; y en consecuencia el establecimiento de la responsabilidad. Estos elementos denotan que bajo este esquema administrativo prevalecen altos niveles de discrecionalidad.

Los principales sujetos de decisión son militantes de partidos políticos y empresarios que se ubican en los niveles más altos del aparato público ${ }^{12}$, logrando de esta manera dominar los procesos de toma de decisión e incluso la selección del personal directivo, tales como ministros, presidentes de empresas públicas, directores y gerentes de distintos niveles, a lo interno de las filas del partido que gobierna (Ochoa et al, 1996).

Formalmente, bajo el esquema burocrático populista, los funcionarios del aparato público son básicamente funcionarios de carrera, cuyos procesos inherentes al reclutamiento, selección, ingreso, remoción, promoción y reclasificación son centralizados, y obedecen, en la generalidad de los casos, al cumplimiento de unas funciones, actividades y tareas previamente descritas en los manuales de clasificación de cargos; el otro tipo de personal que se evidencia son los funcionarios de libre nombramiento y remoción, porque son considerados personal de confianza.

En la práctica, en el reclutamiento y la selección de los funcionarios median los partidos políticos, los cuales llegaron a convertirse en centros de reclutamiento (Ochoa, 2000: 114). Esto introdujo una alta rotación de los cargos, puesto que los partidos políticos debían cubrir en determinados casos promesas políticas asumidas en procesos electorales 0 por acuerdos de tipo político que al fenecer dan paso a la entrada de otros funcionarios, entorpeciendo la rutina y la continuidad administrativa (Córdova, 2000: 133). Este mecanismo de reclutamiento deja al margen el mérito como criterio de ingreso a la administración pública.

La prestación del servicio es una función que corresponde al Estado, se ponen en práctica políticas universalistas y gratuitas, tratando de hacer equitativa la transferencia de los beneficios que se ofrecen a la población, pero no se toma en cuenta la existencia de sectores diferentes y con características heterogéneas; esto no permite tener una precisión sobre los grupos beneficiarios, lo que conduce a una distribución regresiva del ingreso, que favorece a los sectores con mayor capacidad económica.

El financiamiento del servicio descansa en el aparato del Estado, el cual destina recursos de manera desmesurada generando altísimas erogaciones que no se corresponden con los servicios 
prestados, se otorga poca atención a preservar y garantizar el uso adecuado de los recursos. Afirman Pérez y Jaén (1993), que en las etapas de mayor auge y poder económico, el Estado aporta recursos sin establecer ciertas condiciones, y de manera ilimitada, sin atender a la eficiencia, y sin la definición de una política de gastos acorde con los objetivos y metas trazadas. Se trata de solventar los problemas de manera reactiva, lo que conduce al desarrollo de un estilo de proceder en el aparato público de servicios sin calidad.

\subsection{Rasgos del modelo tecnocrático}

El concepto de tecnocracia ${ }^{13}$ es considerado como el término más ambiguo de las ciencias sociales. La designación inicial al término refería al rol que los químicos y físicos asumían en el desarrollo de la sociedad. Posteriormente, fue utilizado para recordar el poder o el influjo de otros tipos de profesionales: el tecnócrata, que viene a representar el director supremo de los procesos de tipo técnico y económico, que corresponde en la empresa pública y privada a los profesionales que buscan el establecimiento de la lógica dirigida al incremento de la eficiencia (Fisichella citado por Bobbio y Mattecuchi, 1988: 1608).

Este modelo se promueve en una economía de mercado basada en la tesis neoliberal, que da paso a la reducción del Estado en el ejercicio de sus funciones, particularmente en el área social que da oportunidad al desarrollo de una política social individualista, inequitativa, y con altos costos.

La extrema división horizontal y vertical del trabajo, altamente compleja, rígida, inspirada en el modelo ideal de Weber, se transforma en estructuras planas, con un mínimo de niveles jerárquicos. Se sustituye en los niveles directivos al hombre de partido por profesionales o técnicos con orientación economicista (Ochoa, 1999). El profesional con capacidad para la dirección y con racionalidad económica, asume tareas de decisión con lo cual contribuye a impulsar el desarrollo científico técnico administrativo, cuestión que ha tenido lugar en nombre de la eficiencia, entendida como el logro de fines a un mínimo costo (Ochoa, 1995).

El proceso de toma de decisión está soportado por la incorporación de los elementos técnicos científicos dirigidos al fin último de rentabilidad y racionalidad económica, disminuyendo el afloramiento de condiciones de tipo política y social dentro de los procesos de decisión.

Según Ochoa (1995: 58-59) los procesos de selección y ascenso son realizados tomando en cuento los méritos, es lo que se ha denominado meritocracia, ésta se constituye en un mecanismo de legitimación de la tecnocracia. La capacidad es el criterio a través del cual se selecciona personal y se asciende en la organización tecnocrática, con lo que se quiere significar que la ubicación de los hombres en los cargos está por encima de intereses personales y extraorganiza-

13 Su aparición en la literatura es a partir del inicio de los años '30 y expandido a partir de los '60 (Ochoa, 1995:15). 
cionales. Cuando Ochoa se refiere a la capacidad, considera no cualquier capacidad sino aquella que contribuye a alcanzar los propósitos y el interés de la dirección de la institución.

En el proceso de reclutamiento de personal aplicado por la tecnocracia sustituye al sistema de mediación de partidos políticos, en este se incorpora de manera recatada la empresa privada, justificada en la transparencia que garantiza en el proceso de reclutamiento, el cual descansa sobre los principios de la meritocracia y la capacidad (Ochoa, 1993: 22; Ochoa, 2000:114).

Bajo esquemas tecnocráticos, la participación directa del Estado en la producción de bienes y servicios se ve reducida, se ponen en práctica la privatización de la administración de áreas importantes de la organización pública, particularmente de las áreas relacionadas con el sector social. La privatización se da con el argumento de contribuir a elevar los niveles de eficiencia de las empresas públicas y de los servicios sociales, porque se piensa que incorporando el estilo gerencial se resuelven los problemas de adecuación entre recursos y atención, estimándose que se puede producir un incremento de la calidad del servicio.

Con la privatización se introducen elementos del mercado como el cobro del servicio, lo que implica "la transferencia de la responsabilidad del financiamiento del sector público al usuario para que éste pague directamente..." (Homedas y Ugalde, 2001). Esto va en detrimento de principios como la gratuidad y la equidad, puesto que un gran número de usuarios, por carecer de recursos ven quebrantado el disfrute de los servicios. Al respecto se- ñalan Homedes y Ugalde (2001) que “...se ha introducido... la privatización cuyo potencial de éxito es dudoso... El experimento puede ser muy costoso... y negativo para la equidad, calidad, eficiencia y satisfacción de los usuarios".

Al respecto señala Montagut (2000: 132), que la privatización está: "asociada principalmente a unas disposiciones según las cuales el gobierno delega... la prestación de los servicios y el cobro de los fondos para financiarlos, esto es... el bien o servicio pasa a regirse por las leyes del mercado, excluyendo o permitiendo su consumo en función de criterios de rentabilidad económica".

\subsection{Rasgos de la Nueva Gestión Pública}

Desde la década de los ochenta, se producen cambios en la administración pública, que han generado un estilo de conducción del aparato público denominado Nueva Gestión Pública (NGP) o modelo postburocrático. Según Barzelay (2001: 11), este paradigma "se origina como un dispositivo conceptual inventado con el propósito de estructurar la discusión académica sobre los cambios contemporáneos en la organización y el gerenciamiento de la rama ejecutiva del gobierno". Asimismo, considera, que "la Nueva Gestión Pública debe ser concebida como un debate, o mejor como un diálogo acerca de cómo enfrentar operativamente cuestiones que se plantean tanto en teoría como en casos concretos, referidos al problema de cómo estructurar, gestionar y controlar la burocracia y el sector público en su conjunto". 
El Centro Latinoamericano de Administración para el Desarrollo (CLAD), elaboró un documento, planteando que, el modelo gerencial tiene su inspiración en las transformaciones organizacionales ocurridas en el sector privado, las cuales modificaron la forma burocrático piramidal de administración, flexibilizando la gestión, disminuyendo los niveles jerárquicos y, por consiguiente, aumentando la autonomía de decisión de los gerentesde ahí el nombre de gerencial (CLAD, 1998).

Se introduce la descentralización hacia los niveles subnacionales, para mejorar las formas de dividir, organizar y coordinar las actividades de los entes $u$ organismos gubernamentales de una manera más horizontal, sin embargo con este nuevo paradigma no se busca sólo la reestructuración organizativa, sino la incorporación de elementos de la administración privada. "Con la descentralización se concede capacidad de decisión a los niveles subnacionales del Estado, de manera que el ciudadano no tiene que recurrir al aparato central" (Ochoa, 1999: 79).

Se flexibilizan las estructuras organizativas, por lo que se presume una toma de decisiones más ágil y oportuna que permite la interacción entre la negociación y la concertación en la adopción de decisiones dando un reconocimiento formal a la participación ciudadana en la gestión y demanda de servicios al aparato del Estado. La toma de decisiones por medio de la participación ciudadana puede estar referida a dos ámbitos distintos: por la definición de los servicios provistos y sus características, y/o sobre la gestión misma de los servicios públicos (Celedón y Noé, 1998: 166).
La NGP tiene como fundamento la Profesionalización de la burocracia, que ejerce funciones en el núcleo de las actividades centrales del Estado, para ello se requiere de aspectos meritocráticos propios del modelo Weberiano (CLAD, 1998). Es preciso destacar que las transformaciones sufridas en el aparato del Estado, dieron paso a la introducción de elementos postburocráticos, que han facilitado la incorporación de lo que Ermida (1999: 139-140), denomina el paradigma de las Relaciones Laborales Postmodernas, cuyas características, son: descentramiento del trabajo, la inestabilidad en el empleo, la individualización y como resultante de lo anterior se produce el abaratamiento del costo de la mano de obra, cuya consecuencia es la reducción del salario real y se refuerza la flexibilización de los horarios en función de las necesidades productivas. Este paradigma, según Ermida (1999) establece dos grupos de trabajadores: a) un núcleo de trabajadores estables, protegido con remuneraciones y calificaciones, y b) una periferia inestable, precaria, rotativa, tercerizada o informal.

Varios tipos de contratación laboral se presentan: a) La contratación del personal fijo o individual, los trabajadores cuentan con derechos laborales individuales más no colectivos, como el derecho a la huelga, a la sindicalización y a la contratación colectiva, lo que se busca es reducir el poder de los trabajadores basado en su acción colectiva. b) Por productividad, los trabajadores son pagados de acuerdo al número de actividades desarrolladas, y seleccionados bajo criterios meritocráticos. 
Según Laurell (2000: 76-77), con esto se busca individualizar el pago y la anulación de la indeminización obligatoria por despido y el monto por antigüedad; y c) utilizando suministradoras de mano de obra, lo que conduce a la desregulación o flexibilización del Derecho del Trabajo. Se establecen contratos precarios de trabajo y subcontrataciones en cascada, caracterizados por una externalización de riesgos y responsabilidades, con reducción de salarios y de empleo (Minayo y da Fonseca, 2000: 274).

Con las reformas instrumentadas por el Estado, se han introducido varias modalidades para prestar los servicios públicos de tipo social, tales como: a) la adjudicación de autonomía de gestión, y b) la asignación de recursos por parte del Estado a través de convenios o contratos de gestión que sean capaces de garantizar el servicio con eficiencia, eficacia y efectividad (CLAD, 1998).

El financiamiento de los servicios sociales, bajo esquemas postburocráticos deja de ser exclusividad del Estado, se incorpora al ciudadano y al sector privado como nuevas fuentes de financiamiento. El ciudadano pasa a convertirse en un usuario-cliente de los servicios, en virtud que debe cancelar tarifas que cubran los gastos y generen niveles de ganancia por los servicios demandados, violando preceptos constitucionales relacionados con la gratuidad de servicios de tipo social ${ }^{14}$, esto arroja como consecuencia que un gran sector de la población quede excluida del disfrute de los servicios por no contar con recursos para cubrir los aportes exigidos.

El propio sector privado interviene en el financiamiento a través de aportes que en ocasiones puede ser en especie o en dinero, ya que ello contribuye a deducir de la renta bruta las liberalidades en cumplimiento de fines de utilidad colectiva y de responsabilidad social como contribuyente, dichos aportes son considerados donaciones que se efectúan a favor del Estado, estas liberalidades deberán perseguir objetivos benéficos, asistenciales, entre otros.

Pues bien, el abordaje de los procedimientos administrativos hasta aquí planteado se constituye en un aspecto fundamental para avanzar en la construcción de conocimiento concerniente al aparato público, por lo que se estudiara su referencia en el nivel subnacional en la prestación de los servicios de salud.

\section{Gestión de los servicios de salud en la Gobernación de Aragua}

En 1995, el gobierno del Estado Aragua estructuró un plan de salud, en el cual se definía como política la "garantía del derecho y acceso a los servicios de salud", planteándose el desarrollo de modelos de gestión y financiamiento en donde se ha dado la generación y manejo de recursos destinados a la prestación de dichos servicios mediante el empleo de distintas alternativas que pretendieron darle autonomía a los hospitales y ambulato- 
rios, para el incremento de su tiempo de funcionamiento y abrir la posibilidad de la venta de los servicios, utilizando la fórmula de la recuperación de costos, la cual ha logrado captar recursos provenientes del sector privado, del propio Estado y de los usuarios.

En este contexto, la gobernación ha adelantado diversos modelos de atención y de gestión que van desde los promovidos directamente por ella, como las fundaciones públicas y aquellos provenientes del interés de la sociedad civil ${ }^{15}$, pero también se ha dado el caso de la existencia de modelos en donde se evidencia la participación de actores sociales y experiencia proveniente de la esfera de la economía social, con lo cual se pone en evidencia el interés por el redimensionamiento y redefinición de la relación Estado-Sociedad. De acuerdo a la diversidad de experiencias que se registran en este Estado, se analizan dos casos como ejemplo de experiencias surgidas y representativas en la realidad histórica, posterior al proceso de descentralización y transferencia de la competencia de los servicios de salud pública.

\subsection{Procesos de decisión en Salud}

La prestación de los servicios de salud pública en Venezuela, ha sido manejada de manera centralizada, con influencia política, marca ausencia de incentivos y mecanismos a la participación ciudadana ${ }^{16}$. En este sentido, analizamos y basándonos en evidencia empírica la forma como se toman las decisiones públicas de salud en la gobernación del Estado Aragua, particularmente en dos centros asistenciales.

El primero de ellos es el Hospital José María Vargas (HJMV), el cual presenta una serie de rasgos propios del modelo tradicional, que se evidenciaran en el desarrollo de este estudio. El organismo responsable de las decisiones esta constituido por un cuerpo colegiado integrado por una Comisión Técnica, encargada de administra el centro asistencial. Se encuentra constituida por los coordinadores de las áreas administrativas y las áreas sustantivas, entre las cuales destacan: Dirección, Administración, Recursos Humanos, Epidemiología, Cirugía, Pediatría, Gineco-Obstetricia. El cargo del director es de libre nombramiento y remo-

15 El concepto Sociedad Civil (SC) ha sido objeto de distintas confrontaciones de tipo teórica que se evidencian en la proliferación de publicaciones donde existen en lengua castellana más de cien trabajos que discuten sobre el término y sus implicaciones, constituyendo el trabajo de Cohen y Arato (2002), editan por primera vez en 1992, una de las obras más significativas donde se discuten numerosas definiciones. Aquí entenderemos por SC, al conjunto de relaciones y transacciones que se establecen entre los ciudadanos de forma desinstitucionalizada en donde priva el carácter económico y social llevada a cabo por un conjunto de actores.

16 Siguiendo a Cunill (1991: 44-48) la PC es entendida como el proceso por medio del cual los individuos en tanto ciudadanos toman parte en alguna actividad pública, en tanto portadores de interese sociales, lo que alude a la intervención de los ciudadanos en el aparato del Estado. 
ción; pues el mismo es personal de confianza del Presidente de Corposalud. En el caso de los coordinadores de las áreas, los mismos son designados en la generalidad de los casos de manera discrecional por el Director Municipal ${ }^{17}$ de Salud previa aprobación del Presidente de CORPOSALUD (Picos, 2003). Esta manera de designación revela la puesta en práctica de criterios políticos clientelares como principio de selección para ejercer los cargos de la administración pública en salud. La designación de los coordinadores y del director se realiza todos los años con posibilidades de redesignación; dado que son funcionarios de libre nombramiento y remoción.

La Comisión Técnica efectúa reuniones cada quince días para programar actividades operativas en las cuales se identifican problemas, se trazan las alternativas de solución y se implementan las soluciones; y otras reuniones mensuales para evaluar los efectos y la eficiencia de la ejecución de las alternativas seleccionadas.

Existe una Junta Socio-Sanitaria, que participa en la identificación de los problemas del centro asistencial, de manera conjunta con la Comisión Técnica (Picos, 2003). Dicha Junta formalmente es una instancia encargada de supervisar y dirigir las actividades administrativas del Hospital, así como adelantar prácticas de auditoria operativa, cuyo propósito es revisar los libros, el control de bienes e inventarios, la contabilidad fiscal y administración de los recursos humanos. Pero, la capacidad resolutiva de los miembros ${ }^{18}$ de la Junta Socio-Sanitaria, es limitada por cuanto el manejo de los asuntos relacionados con la auditoria, requiere de un nivel de conocimiento y manejo de aspectos técnicos, que sus integrantes no poseen.

Con todas estas actividades se da paso, en lo formal a la participación de la comunidad en los procesos de toma de decisión en el hospital; pero, en este caso en particular, la práctica demuestra que se encuentra al margen del proceso sistemático de decisión, ya que la participación de los representantes de la junta, se centra en el mero planteamiento de sus problemas y de las posibles alternativas de soluciones; sin embargo, la última palabra recae en el director del hospital, sin considerar la opinión de los miembros de la Junta Socio-Sanitaria; el director es la máxima instancia de decisión, pues se restringe el paso a un debate o examen que permita generar opciones diversas y realizar una evaluación objetiva. Esto denota una toma de decisión centralizada en la cúspide del poder con alto nivel de discrecionalidad, lo que dificulta la democratización del poder en los servicios de salud.

17 A partir de 1998, la configuración sanitaria del Estado entró en plena correspondencia con la división político territorial, lo que dió paso a la instauración del cargo de Director Municipal de Salud.

18 La Junta esta conformada por el director, el subdirector, además de un representante del personal del hospital; asociaciones de vecinos, cooperativas, empresas, comercio, clero, sociedad de padres y representantes, asociaciones civiles o fundaciones. 
El segundo caso seleccionado, es el Hospital Estadal Los Samanes, administrado por la Fundación Materno Infantil Los Samanes, que nace como modelo "coparticipativo", entre el Estado y la sociedad civil, siendo propuesto por el propio ejecutivo regional, con el propósito de traspasar las funciones de salud a la sociedad civil mediante documento en el que se garantiza el apoyo financiero del ejecutivo regional al Hospital (Zárraga, 2001).

Para la puesta en práctica de esta iniciativa regional, el gobernador del Estado convocó a la Fundación Distrital Materno Infantil del Estado Aragua, hoy Fundación Estadal Los Samanes para encomendarle la puesta en funcionamiento de un ambulatorio urbano, cuya instalación estaba abandona. Fue dotada de equipos, mobiliario e insumos por parte de la gobernación del Estado Aragua y entregadas a la Fundación para dirigir los servicios (Zárraga, 2001). Esto permitió el inicio del funcionamiento de un hospital materno infantil, bajo la dirección de un grupo de quince personas denominados miembros fundadores, quienes en la actualidad integran la asamblea del hospital, y entre los cuales destacan como miembros principales el Gobernador del Estado, el Presidente de CORPOSALUD el Presidente-Director del hospital, y doce personas más. Fortuitamente esta experiencia se adelanta en el contexto de la descentralización como política pública.

El cuerpo colegiado está representado por una Junta Directiva, integrada por el presidente-director quien desde hace quince años fue designado por el gobernador, a los fines de que éste como empresario exitoso de la región transfirie- ra su experiencia de gestión a este hospital, justificando su presencia con el discurso de ser éste un representante de la "sociedad civil" -condición esta que se ha mantenido hasta el momento de efectuarse esta investigación-, un vicepresidente (primera dama del ejecutivo regional), secretario, un tesorero y el cuerpo de directores de las áreas sustantivas y administrativas, todos tiene derecho a redesignación cada dos años en el ejercicio de su cargo (Hospital Los Samanes, 2003).

La Junta Directiva esta integrada por once miembros fundadores; de ellos solamente la persona que ocupa el cargo de vicepresidente ha cambiado, pues el mismo recae en la figura de la primera dama del gobierno regional; el resto; es decir, cuatro directivos Médicos, un secretario y cuatro sujetos no profesionales, se han mantenido invariable en el tiempo. Estos fueron designados bajo criterio político, pues se requerían personas vinculadas a la gestión, al proyecto y al interés de avanzar en la descentralización; y criterios tecnocráticos, dada la presencia de una "ideología economisista, pues se priorizó el interés económico por encima de cualquier cosa" (Ochoa, 1993: 19).

Una razón que explica el mantenimiento de los miembros en esta Junta Directiva por espacio de dieciséis años, obedece al hecho que diez de sus integrantes son a su vez integrantes de los quince miembros que conforman Asamblea. Se evidencia que entre estas dos instancias no hay un proceso independiente y autónomo, que albergarse un fenómeno poco transparente con respecto al proceso de decisión, pues el Presidente-Director es el que concentra las decisiones, y además se encarga de informar 
mensualmente al resto de los miembros de la Junta Directiva las actividades a ser realizadas.

Con respecto a las decisiones relacionadas con la política de recuperación de costos, servicios complementarios para la salud, personal, mantenimiento, equipos, insumos, suministros, es el propio presidente-director quien toma las decisiones, sin que estas sean el resultado de un proceso amplio de consulta (Quiróz, 2004). Por lo cual estamos ante la presencia de un modelo modernizante autoritario.

Presumimos que la designación de miembros de la "sociedad civil" en la dirección de un centro como este, se dio con el propósito de avanzar en la descentralización; sin embargo, la forma en la cual se han organizado las instancias decisionales aluden a un proceso de toma de decisión altamente centralizado en la cúspide del poder de esta institución, por lo que ni se registra la participación de los ciudadanos ni tampoco se han dado los mecanismos que permitan un proceso de decisiones democratizador. Lo que lleva a pensar que se ha formado una instancia de decisión encarnada en una figura personalista, arbitraria, autoritaria representada en el presidentedirector, en un contexto donde se privilegia el discurso de la descentralización, pero que en la realidad no se corresponde con el desarrollo del proceso que se ha venido dando en el Hospital los Samanes.

\subsection{Administración de Personal en Salud}

El régimen laboral reviste una gran importancia para el funcionamiento de los servicios de salud pública, ya que se conjugan actividades de uso intensivo de mano de obra. Una gran mayoría de los profesionales de la salud están empleados en el sector público, cuyas condiciones de trabajo se negocian entre los sindicatos y los entes subnacionales.

Analizando los criterios de selección, así como los mecanismos de contratación del personal en los casos de estudio, se tiene que en el HJMV, se evidencia la presencia de funcionarios de carrera, entre los cuales se encuentran: personal administrativo, profesionales de la salud y obreros ${ }^{19}$. Analizado bajo las características del modelo burocrático populista, dan cuenta de la existencia de un sistema de reclutamiento, selección, ingreso, remoción, promoción y reclasificación, donde sistemáticamente se ha encontrado la presencia del "amiguismo", "compadrazgo" y el "clientelismo político", lo que no ha impedido que los funcionarios, en la generalidad de los casos cumplan con las funciones, actividades y tareas que se encuentran previamente descritas en los manuales de clasificación de cargos de la administración pública. Se registra; además, la presencia de personal médicos y paramédicos, seleccionados mediante concurso de credenciales, es decir por criterios meritocráticos propios de los modelos Weberiano y Tecnocrático; con esta práctica se tiende a minimizar el efecto del clientelismo político.

Con respecto a los funcionarios de carrera, fueron contratados para periodos de tiempo indeterminados, los cuales 
gozan de remuneración por el cargo que desempeñan; disfrute de vacaciones; bonificaciones de fin de año; jubilaciones; prestación de antigüedades, fideicomiso, derecho a la sindicalización; huelga, contratación colectiva; entre otros ${ }^{20}$.

En relación con el personal contratado, estos permanecen por períodos de tiempo determinado, no gozan de los mismos derechos y beneficios que los funcionarios de carrera por lo que su relación laboral se ve afectada, entre otros aspectos porque no gozan de derechos colectivos como la huelga, la sindicalización, jubilación, entre otros.

Estos mecanismos de contratación evidencian, dos grupos de trabajadores, tal y como lo señala Ermida (1999), los estables y aquellos que se encuentran en la periferia, lo que demuestra la puesta en práctica de manera embrionaria de rasgos de la NGP en materia de administración de personal.

A diferencia del Hospital José María Vargas, en el caso del Hospital Los Samanes, tanto el personal administrativo, técnico asistencial, son seleccionados con base a tres criterios que aluden al promedio ponderado de calificaciones; los requerimientos exigidos para el desempeño del cargo; $y$, vivir cerca del Hospital (Quiróz, 2004). Los dos primeros criterios dan cuenta de la utilización del mérito como mecanismo de ingreso a las filas del aparato público de salud a nivel subnacional, propio de la organización tecnocrática; y el último criterio tiene como propósito evitar los riesgos de la ausencia y/o retraso al sitio de trabajo.

Una vez seleccionados los sujetos, son sometidos al proceso de inducción en el que se comunica: visión; misión; objetivos; los servicios que presta el Hospital; y las normativas a seguir por parte del personal de planta, suplentes y los que se encuentran en períodos de prueba; en este último caso, se les contrata por lapsos que oscilan, según Quiroz (2004), entre seis meses y dos años; durante ese tiempo gozan de derechos individuales, como por ejemplo: el Programa de Alimentación, el cual se otorga a través de concesiones de una comida balanceada por jornada laboral; pago del fideicomiso de la prestación de antigüedad de manera mensual; entre otros (Garay, Art. 108 de LOT).

Cuentan con una modalidad de seguro social denominado Sistema de Autoliquidación de la Empresa, que se distancia de lo planteado en la Ley del Seguro Social, porque adopta un sistema propio, que es definido por ellos "como un novedoso sistema de liquidación, de seguridad social a los empleados..." (Hospital Los Samanes, 2003). Esto es indicativo de que con la implantación de este sistema se va en contra de lo planteado en la Ley Orgánica del Trabajo; primero, porque un período de prueba no debe exceder de tres meses, ya que posterior a este lapso de tiempo el patrono está en la obligación de contratar al trabajador; y

20 Los derechos laborales se encuentran consagrados en La Constitución de 1999 (Asamblea Nacional Constituyente, 2000), Ley Orgánica de Trabajo (Congreso de la República, 1997), Ley sobre el Estatuto de la función Pública (Presidente de la República, 2001). 
segundo, porque han abierto un régimen de seguridad social distinto al planteado en la Ley vigente.

En síntesis, la modalidad de contratación del personal en estos dos centros asistenciales del Estado Aragua, deja ver la puesta en práctica de algunos rasgos del enfoque de la NGP, pues se introducen mecanismos que tienden a la flexibilización y subsecuente precarización de la relación laboral.

\subsection{Prestación de los Servicio de salud}

La prestación de los servicios de salud está condicionada por las características que adquiere la oferta que se hace en los distintos establecimientos de salud; hace referencia entre otras variables a: cantidad, calidad, organización, financiamiento, distribución espacial, niveles tecnológicos, infraestructura y diversidad de las especialidades; y por el lado de la demanda, relacionada con factores sociodemográficos, culturales de la población y sobre todo los de índole epidemiológica. En los casos de estudio, se privilegian los aspectos que se describen a continuación.

En el HJMV, según Picos (2003), el servicio es prestado por orden de llegada y de manera gratuita, se brinda una atención preventiva por medio de los programas de vacunación, infecciones de transmisión sexual, SIDA; lactancia materna; programa de control de cáncer de cuello uterino; control prenatal. Al mismo tiempo, se prestan servicios preventivos del I y II nivel de atención, el primer nivel tiene un enfoque integral de salud que combina la prevención primaria y secundaria, el segundo nivel presta atención especializada, entre los servicios destacan: Medicina Interna, Pediatría Cirugía General y Ginecología-Obstetricia, Odontología y Cirugía Ambulatoria, Hospitalización y Laboratorio, Rayos X y aplicación del Modelo de Atención Integral desde el 2000 (Picos, 2003). Fundamentalmente se presta atención a los miembros de las áreas aledañas al Hospital, que son aproximadamente 100.000 habitantes (Picos, 2003).

En el caso del Hospital Los Samanes, el servicio es prestado por orden de llegada o por previa cita, y se impone a toda persona la carnetización para el disfrute del servicio quien llega directamente a una caja para pagar la consulta o el servicio requerido, resquebrajándose así el principio de gratuidad consagrado en la Constitución de la República Bolivariana de Venezuela (1999). Se brinda una atención preventiva a través de los siguientes programas: Inmunizaciones, Atención al Adulto, Lactancia Materna y Control Prenatal (Zárraga, 2001).

Adicionalmente se prestan servicios curativos entre los que destacan: Emergencia Pediátrica, Consulta Externa, Neumonología, Nefrología, Pediatría, Dermatología, Traumatología, Ortopedia, Oftalmología, Odontología, Ginecología Obstétrica, Otorrinolaringología, Cardiología General y Pediátrica, Cirugía Plástica Pediatría y Puericultura, Neurología Pediátrica, Nefrología, Medicina General y Medicina Interna, Laboratorio y Rayos X, Bacteriología, Ultrasonido. Entre otros servicios está la sala de cura, sala de rehidratación, cirugía general y pediátrica, hospitalización, historias médicas, farmacia, comedor, cafetín, biblio- 
teca, auditorio, galería de artes, óptica y ortopedia (Zárraga, 2001; Hospital Los Samanes, 2001 y 2003). La atención se brinda a diversos sectores de la población ${ }^{21}$ de Aragua, y a los Estados del centro del país (Hospital Los Samanes, 2001).

Los servicios prestados evidencian que a pesar, de mencionarse programas de corte preventivo, las acciones llevadas a cabo en estos dos establecimientos de salud, están signadas por el predominio de las actividades de tipo curativo, y que son adelantadas con regularidad y fluidez en el otorgamiento de las citas. El predo- minio de atención curativa se ha convertido en un modelo de asistencia a la enfermedad, erigiéndose como el vértice principal de la ciencia médica, la cual se encarga del estudio y control de la enfermedad que padece un organismo biológico que amerita ser intervenido y modificar la condición patogénica mediante el empleo de agentes químicos, físicos, quirúrgicos, entre otros.

A continuación se establece un cuadro comparativo de los servicios preventivos y curativos, ofrecidos en los dos centros salud estudiados en la gobernación del Estado Aragua.

\section{Cuadro 1. Gobernación del Estado Aragua Servicios ofrecidos en el Hospital José María Vargas y en el Hospital Los Samanes}

\section{Servicios ofrecidos}

Preventivos

Curativos/

Especializados

\section{Centros Asistenciales}

Hospital José María Vargas

\begin{abstract}
Programas de Vacunación, Infecciones de Transmisión sexual, SIDA; Lactancia Materna; Programa de Control de Cáncer de Cuello Uterino; Control Prenatal
\end{abstract}

Medicina Interna, Pediatría Cirugía General y Ginecología-Obstetricia, Odontología y Cirugía Ambulatoria, Hospitalización y Laboratorio, Rayos X
Inmunizaciones, Atención al Adulto, Lactancia Materna y Control Prenatal

Emergencia Pediátrica, Consulta externa, Neumonología, Nefrología, Pediatría, Dermatología, Traumatología, Ortopedia, Oftalmología, Odontología, Ginecología Obstétrica, Otorrinolaringología, Cardiología General y Pediátrica, Cirugía Plástica Pediatría y Puericultura, Neurología Pediátrica, Nefrología, Medicina General y Medicina Interna, Laboratorio y Rayos X, Bacteriología, Ultrasonido

Fuente: Elaboración propia sobre la información suministrada en los respectivos hospitales.

21 Las personas que no se encuentran residencias cerca del Hospital, acuden al Hospital Central de Maracay (Maracay es la capital del estado Aragua). Incluso, quienes acuden al Hospital Los Samanes y no cuentan con recursos para cancelar los servicios se dirigen al mencionado centro asistencial. 
Como puede ser apreciado en ambos casos predominan acciones de tipo curativo, lo que es indicativo que a pesar de haberse ensayado modelos de gestión con algunas características diferenciales, en el caso de la prestación del servicio, lo curativo sigue ocupando la principal forma de atención. En ninguno de estos dos centros de salud, se pudo hacer evidente el establecimiento de pautas que indicaran trabajo en la comunidad, más que algunas iniciativas muy esporádicas de charlas e información sobre salud, con lo que se refuerza la tesis que las acciones de índole preventiva son muy pocas.

\subsection{Fuentes del Financiamiento de los servicios de Salud}

La identificación de las fuentes de financiamiento ${ }^{22}$ conduce a determinar el grado de compromiso que tienen los distintos actores con relación al funcionamiento de un sector. En el caso específico del Estado de Bienestar, más que altruismo, solidaridad, beneficencia o caridad, el haber asumido en forma exclusiva la responsabilidad del financiamiento de la salud ha sido interpretado por algunos autores como que el Estado de Bienestar fue una manera de contrarrestar en Occidente el avance del comunismo, para otros consiste en darle cabida a la dinámica de las relaciones de tipo capitalista y según otros autores se trata solventar los conflictos políticos que cerraban la consolidación de una democracia nacional (Montagut, 2000 y Ochando, 2002).

Sin embargo, es preciso señalar, que la tendencia neoliberal impuesta por los organismos multilaterales; principalmente el Banco Mundial, ha sido el financiamiento de reformas que apuntan a reducir el gasto por parte del Estado a través de la introducción de la lógica del mercado, imponiendo el financiamiento a los ciudadanos quienes dejan de ser meros ciudadanos para convertirse en clientes que pagan por la obtención del beneficio de un servicio que ha sido elegido por ellos. Esta lógica impone limitaciones sociales, puesto que afecta la posibilidad de acceder a un servicio de salud en términos económicos y de acuerdo a las necesidades del usuario; asimismo, se imponen restricciones en términos de igualdad; acceden al servicio quienes cuentan con recursos para hacerlo y los que no, están limitados de la lógica del disfrute del servicio (Rincón, 2004).

Para Capriles et al (2001) y Gamboa (2003), el financiamiento asume un rol esencial en la forma como funcionan los sistemas de salud, cuyo análisis debe tomar en cuenta aspectos como volumen, origen, administración y destino, pero también: “... el proceso de financiamiento tiene un carácter determinante para el sistema de salud... fija los límites dentro de los cuales dicho sistema puede ser más eficaz y eficiente en términos de tándonos a la simple identificación de las fuentes de financiamiento y a los rubros de gastos. 
sus resultados finales, es decir, en relación con los impactos sobre la salud de la población, expresados en las modalidades de un cierto perfil epidemiológico" (Capriles et al, 2001: 2-3).

Por su parte, señala Gamboa (2003: 164 y 166) que el origen de los fondos y el modelo de administración de los mismos resultan determinantes respecto al destino final de los recursos y sus resultados. El volumen de los fondos es casi inseparable del origen de los mismos puesto que existe una relación inversamente proporcional entre el gasto público y el privado, de tal forma que el gasto indica la naturaleza del sistema de salud.

En el caso del Estado Aragua, el financiamiento de los servicios de salud proviene de varias fuentes; sin embargo, en el HJMV, la principal fuente de financiamiento recae en la Gobernación del Estado, a través de Corposalud, ente que percibe un presupuesto asignado por vía del Ministerio. Esto demuestra un "financiamiento a la oferta de servicios -típica de los servicios públicos nacionales de salud-" (Gamboa, 2003: 166).

Estos aportes del Estado están orientados particularmente a sufragar gastos de funcionamiento, entre los que se menciona: adquisición de insumos, suministros y equipos, mantenimiento de la infraestructura hospitalaria (física, equipos médicos, muebles, entre otros) y para el pago del personal que se encuentra bajo de contratos (Picos, 2003).

\section{En el Hospital Estadal Los Sama-}

nes, se evidencia un financiamiento mixto; la principal fuente de financiamiento descansa en la gobernación, la cual aporta el mayor porcentaje de los ingresos del Hospital, que en palabras de Quiróz
(2004) asciende a ocho millardos de bolívares. La otra fuente esta constituida por los usuarios a través del Programa de Recuperación de Costos.

Existe una oficina de atención al público cuya finalidad principal es "ayudar" a aquellas personas que por una $u$ otra razón soliciten exoneración parcial o total de algún servicio, sin previo estudio social (Hospital Los Samanes, 2003). Sin embargo, no tiene sentido el estudio social, cuando en la práctica según palabras de Quiroz (2004), "muchas personas de las que se les exonera se les dice, yo se lo voy a exonerar pero si vuelve tiene que colaborar...esa persona viene y cancela", pero al regresar para solicitar nuevamente el servicio y no cancela en su debida oportunidad, no tiene derecho a contar con el servicio, en este centro asistencial, pues en la historia clínica se le indica que el mismo esta en mora, con lo que automáticamente se el restringe el acceso al servicio y debe acudir a otro centro asistencial de la región.

Los ingresos provenientes por parte de la gobernación y de los usuarios, son utilizados para el pago del personal, adquisición de materiales médicos, quirúrgicos, odontológicos, laboratorio, radiológicos, oficina, mantenimiento, mobiliario y lencería, imagen corporativa, equipos tecnológicos, entre otros.

El financiamiento es uno de los aspectos que marca la pauta en el funcionamiento de la prestación de los servicios de salud, cuyo grado de contribución al mejoramiento en la atención puede ser seriamente cuestionable, ya que no necesariamente se constituye en factor determinante para garantizar per sé la calidad del servicio, pues la complacencia o 
satisfacción del usuario por la atención recibida puede ser vulnerable cuando debe cancelar por el servicio.

Las fuentes de financiamiento dan cuenta del tradicional financiamiento gubernamental; $y$ los aportes de los ciudadanos, a través del pago en dinero, con esta lógica el Estado aprende a vender sus servicios y ha incorporar elementos del mercado, obviando las imperfecciones que esto implica, pues el ciudadano pasa a ser tratado como cliente; anteponiéndose las necesidades mercantiles en contraposición a los derechos ciudadanos de gratuidad del servicio, ocasionando problemas en la cobertura y accesibilidad de la población a los servicios de salud.

\section{Conclusiones}

En la década del noventa las gobernaciones se vieron favorecidas por el auge adquirido por la descentralización, lo cual permitió que se emprendieran procesos de transferencia de competencias al nivel subnacional. En las gobernaciones se crearon instancias que permitieron adelantar políticas regionales en salud, como CORPOSALUD en el Estado Aragua.

La transferencia de la competencia de salud a los niveles subnacionales, hizo que las gobernaciones adoptaran diversas modalidades de gestión que oscilan entre la promoción directa del Estado, creación de fundaciones públicas; fundaciones corporativas, entre otras. Un ejemplo de ello lo constituye la gobernación del Estado Aragua, a través del Hospital José María Vargas, es administrado directamente por el gobierno regional; $y$ el Hospital Estadal los Samanes por una fundación pública.
Estos han desarrollado procesos administrativos que dan cuenta de una toma de decisiones centralizada en la cúspide del poder, con influencia partidista, lo que evidencia rasgos burocráticos populistas. La administración de personal se ciñe bajo esquemas de selección por méritos, tal y como lo refiere Weber en su propuesta de organización, y el modelo de tecnocracia. Con relación a los mecanismos de contratación se evidencia la presencia de funcionarios públicos contratados para períodos indeterminados y los contratados a tiempo determinado, lo que da paso en este último caso a la introducción de procesos que flexibilizan y precarizan las relaciones laborales.

En la generalidad de los casos la política de salud esta vinculada con acciones de tipo curativo. Con la descentralización se comenzó un proceso con el que se da inicio y ampliación a la privatización de los servicios de salud, al propio tiempo que variadas experiencias de gestión se pusieran en marcha, dada la necesidad perentoria de buscar respuestas a los problemas que se presentaban, como el déficit de recursos económicos, abriéndose así espacios para la búsqueda de alternativas de financiamiento, por lo que se pone en práctica el cobro de los servicios de salud, lo que implicó la transferencia de la responsabilidad del financiamiento del sector público a los usuarios, esto en el caso venezolano está en franca contradicción con los preceptos de gratuidad establecidos en la Constitución de la República Bolivariana aprobada a finales de la década de los noventa. 


\section{Referencias Bibliográficas}

Asamblea Nacional Constituyente. Constitución de la República Bolivariana de Venezuela. Gaceta Oficial Gaceta Oficial No. 5.453, de fecha 24 de marzo de 2000. Caracas.

Baezelay, Michael (2001), "La Nueva Gestión Pública. Una invitación al diálogo globalizado". En: seminario de la London School of Economics and Political Science. Paris, France.

Bobbio, Norberto y Mattecucci, Incola (1988), Diccionario de Política. Siglo veintiuno Editores. DF. México.

Capriles, Edgar, Díaz Polanco, Jorge, Fernández, Yajira y Pereira Cegrí (2001), "La Reforma del Sistema de Salud de Venezuela: Opciones y Perspectivas". En Díaz Polanco, Jorge (Coord.). La Reforma de Salud en Venezuela: Aspectos Políticos e Institucionales de la Descentralización de la Salud en Venezuela. CENDES, CIID, MSDS, CORPO SALUD. IESA, FUNDACIÓN POLAR. Caracas.

Celedón, Carmen y Noé, Marcela (1998), "Participación y Gestión en salud”. En CLAD. OPS/OMS (1998). Hacia la implantación de modelos de administración gerencial en salud pública. XII concurso de ensayos del CLAD, ensayos premiados. Washington.

CLAD (1998), Una Nueva Gestión Pública para América Latina. Caracas.

Cohen, Jean y Andrew, Arato (2000), Sociedad Civil y Teoría Política. FCE. México.

Congreso de la República de Venezuela (1989), Ley Orgánica de Descentralización, Delimitación y Transferencia de competencias del Poder Público. Gaceta Oficial Gaceta Oficial No. 4.153, de fecha 28 de Diciembre. Caracas.
Congreso de la República de Venezuela (1997). Ley Orgánica del Trabajo. Gaceta Oficial Extraordinaria 5152, 19 de junio. Imprenta Nacional. Caracas.

Garay, Juan (2000), Legislación Laboral. Práctica. Ley Orgánica del Trabajo. Ediciones Juan Garay. Caracas, Venezuela.

Córdova, Edgar (2000), "La flexibilización laboral en los entes descentralizados de competencias exclusivas". En Revista Venezolana de Ciencias Sociales. Vol. 4. No. 2. Cabimas. Venezuela, pp. 129-145.

Cunill, Nuria (1991), Participación Ciudadana. Centro Latinoamericano de Administración para el Desarrollo. Caracas.

Dirección Regional de Salud del Estado Aragua (2000), Establecimiento de salud del Ministerio de Salud por municipios y tipos. En www.msds.gov.ve. Fecha de consulta 02-07-2002.

Ermida, Oscar (1999), "Globalización y Relaciones Laborales". Revista Venezolana de Gerencia. Año 4. No. 9. Universidad del Zulia. Maracaibo, Venezuela. Pp. 135-148.

Gamboa, Teresa (2003), Modernización de los Sistemas de Salud en Colombia y Venezuela. Hacia un cambio en las relaciones de poder. Tesis Doctoral en Ciencia Política. Universidad del Zulia. Maracaibo. Venezuela.

Gobernación del Estado Aragua (1995), Proyecto salud. Plan Estadal de salud del Estado Aragua. Maracay, Venezuela.

Hernández, Sampieri; Fernández, Carlos y Baptista, Pilar (1991), Metodología de la Investigación. McGraw-Hill. 1 era Edición México.

Homedes, Nuria y Ugalde, Antonio (2001), "Privatización de los servicios de salud: las experiencias de Chile y Costa 
Rica". En www.clad.org.ve. Fecha de Consulta 20-05-2002.

Hospital Los Samanes (2003), Historia, Objetivos y Reglamento. Maracay, Estado Aragua.

Hospital Los Samanes (2001), Revista El Samán. Año 8. No. 01. Edición 39. Estado Aragua. Maracay, Venezuela.

Laurell, Asa Cristina (2000), "Globalización, Políticas Neoliberales y Salud". En Briceño, R, De Souza Minayo, M. y Coimbra, C (coord.). Salud y equidad: una mirada desde las Ciencias Sociales. Editora FIOCRUZ. Caracas.

Málaga, Hernán; Manzanilla Luis y Toba, María (1996), Proyecto "Municipios Hacia la Salud. Experiencia Venezolana". OPS-OMS-MSAS, Caracas, Venezuela.

Minayo, Carlos y Da Fonseca, Sonia (2000), "Descentralización y Participación en Salud: nuevos partes para al discusión". En Briceño, R., De Souza Minayo, M. y Coimbra, C. (coord.). Salud y Equidad: una mirada desde las Ciencias Sociales. Editora FIOCRUZ. Caracas.

Montagut, Teresa (2000), Política Social. Una introducción. Editorial Ariel. Barcelona. España.

Ministerio de Salud y Desarrollo Social (MSDS) (2001), República de Venezuela. Decreto del Modelo de Atención Integral. No. 016 del 14 de Enero. Gaceta Oficial No. 36.876. Caracas, Venezuela.

Ochando, Carlos (2002), El Estado del Bienestar. Editorial Ariel. Barcelona, España.

Ochoa, Haydée (1993), "Consideraciones en torno al fenómeno de la Tecnocracia”. En Cuestiones Políticas. No. 11. Universidad del Zulia. Venezuela. pp. 17-39.
Ochoa, Haydée (1995), Tecnocracia y Empresas Públicas en Venezuela (1948-1991). EDILUZ. Universidad del Zulia. Venezuela.

Ochoa, Haydée (1996/1997), "La Reforma de la Administración Pública en Venezuela -Proyectos y Realidad-". En Gestión de Políticas Públicas. Nos. 7-8. España. pp. 177-188.

Ochoa, Haydée (1999), "Elementos teóricos para el estudio de la modernización administrativa de las gobernaciones en Venezuela". En Revista Venezolana de Ciencias Sociales. Vol. 3. No. 1. Cabimas. Venezuela, pp. 69-91.

Ochoa, Haydée (2000), "Modernización de la administración de personal en el aparato público venezolano". En Revista Venezolana de Ciencias Sociales. Vol. 4, No. 2. pp. 107-128.

Ochoa, Haydée; Valladares, Mirtha y Rodríguez, Isabel (1996), "Administración pública y populismo en Venezuela. En Revista Venezolana de Gerencia. Vol 1. No. 1. Universidad del Zulia. Venezuela. pp. 39-58.

Pérez, Auristela y Jaén, María Helena (1993), "Descentralización de la salud: por una atención de calidad". En De La Cruz, Rafael (coord.). Descentralización, Gobernabilidad, Democracia. COPRE; PNUD, Nueva Sociedad. Caracas.

Presidente de la República (2001), Decreto con Fuerza de Ley Sobre el Estatuto de la Función Pública. Gaceta No. 5557, marzo 13. Imprenta Nacional. Caracas.

Rincón Becerra, María T. (2004), Modelos de Gestión de los servicios de salud promovidos por las gobernaciones venezolanas (1989-2003). Trabajo Final del Programa de Investigador Novel. LUZ-FONACIT. Maracaibo, Venezuela. 
Rodríguez, Isabel (2000), "Descentralización en Salud. Década de los 90. Marco normativo en cinco Estados venezolanos". En Revistas de Ciencias Sociales Vol. VI. No. 2. LUZ. Maracaibo, Venezuela.

SISOV (2005). Indicadores de Salud en Venezuela. En www.sisov.mpd.gov.ve, fecha de consulta 01/11/2004.

Tablante, Carlos (1993), La Descentralización: Un triunfo del ciudadano. Descentralizar para avanzar. Gobierno de Aragua.

Weber, Max (1976), Economía y Sociedad. Esbozo de Sociología Comprensiva. Tomo II. 2da reimpresión en español. Fondo de Cultura Económica. México.

Zárraga, Pedro (2001), 20 Experiencias Exitosas en Gestión de Salud Pública. Fundación Polar, Caracas. Venezuela.

\section{Entrevistas}

Picos, Victor (2003), Modelo de Gestión del Hospital José María Vargas, Entrevista Personal. Director. 22 de Julio, Maracay. Estado Aragua. Entrevista realizada por Deyanira Henríquez.

Quiróz, Freddy (2004), "Modelo de Gestión del Hospital Estatal Los Samanes", Entrevista Personal. Jefe del Departamento de Recursos Humanos, 30 de Enero. Maracay, Estado Aragua. Entrevista realizada por María T. Rincón Becerra y Deyanira Henríquez.

Ramos Jiménez, Alfredo (1995), Los partidos políticos en las democracias latinoamericanas. Consejo de Publicaciones de la Universidad de Los Andes. Mérida-Venezuela. 